\title{
Doppler redistribution of anisotropic radiation and resonance polarization in moving scattering media
}

\section{Predicted results for the coronal O VI $1032 \AA$ line}

\author{
S. Sahal-Bréchot ${ }^{1}$ and N.-E. Raouafi ${ }^{2,3, \star}$ \\ 1 Laboratoire d'Etude du Rayonnement et de la Matière en Astrophysique (LERMA), Observatoire de Paris-Meudon, 92195 Meudon, France \\ e-mail: sylvie.sahal-brechot@obspm.fr \\ 2 Max-Planck-Institut für Sonnensystemforschung, 37191 Katlenburg-Lindau, Germany \\ 3 National Solar Observatory, PO Box 26732 Tucson, AZ 85726-6732, USA
}

Received 4 February 2005 / Accepted 11 June 2005

ABSTRACT

\begin{abstract}
The present paper puts the Doppler redistribution theory of anisotropic radiation and resonance polarization in moving scattering media into a predictive application following Sahal-Bréchot et al. (1998, A\&A, 340, 579; $\mathrm{P}_{\mathrm{II}}$ ), using the example of the O VI $1032 \AA$ line of the corona. Numerical results are presented for different heights over the limb compatible with observations of different instruments on SOHO (SUMER, CDS, UVCS) but far from coronal holes in order to avoid inhomogeneities of the transition zone incident radiation. The orders of magnitude of our numerical results confirm the theoretical predictions of $\mathrm{P}_{\mathrm{II}}$ and show that interpretation of polarimetric data of the O VI $1032 \AA$ line of the corona, associated to the shift and the Doppler-dimming effect, offers a powerful diagnostic method of the complete velocity field vector, provided that the partial anisotropy of the incident radiation field be taken into account. These results also explain the precision of the diagnostic of interpretation polarimetric observations achieved by the ultraviolet spectrometer SUMER in the south polar coronal hole at $270^{\prime \prime}$ above the solar limb; e.g. the solar wind acceleration region.
\end{abstract}

Key words. atomic processes - line: formation - line: profile - polarization - Sun: corona - Sun: solar wind

\section{Introduction}

In the light of recent developments in the theory of matterradiation interaction in the presence of a magnetic fieldas applied to non-LTE spectropolarimetry in astrophysics, we revisited the theory of anisotropic resonance line scattering in moving media by means of the density-matrix formalism, which was introduced in a previous paper (Sahal-Bréchot et al. 1998 , hereafter referred to as $\mathrm{P}_{\mathrm{II}}$ ). There we extended the theory of the "Doppler dimming" effect (Gabriel 1971; Kohl \& Withbroe 1982) to resonance polarization. The purpose of $\mathrm{P}_{\mathrm{II}}$ was to develop a theoretical method of determining the matter velocity field vector in moving scattering media by means of a spectropolarimetric diagnostic.

In $\mathrm{P}_{\mathrm{II}}$ we first rederived the basic theory of resonance scattering in the density matrix formalism (Cohen-Tannoudji 1977; Blum 1981; Cohen-Tannoudji et al. 1988) for a partially directive and frequency-dependent incident radiation, the moving atoms having an anisotropic velocity distribution (a Maxwellian distribution of velocities with a drift velocity

^ Associated researcher to the LERMA Department, Observatoire de Paris-Meudon, France. vector). In fact, the density-matrix formalism is well adapted to polarimetric studies. Second, the theory developed in $\mathrm{P}_{\mathrm{II}}$ not only showed that the re-emitted line is shifted and its intensity dimmed by the Doppler effect, due to the matter velocity field of the plasma, but also predicted that its two linear polarization parameters, degree and direction of polarization, are sensitive to the matter velocity field vector.

The theory was then applied to a two-level atom in order to obtain analytical expressions for the Stokes parameters of the scattered line. The O VI $1032 \AA\left(2 \mathrm{p}{ }^{2} \mathrm{P}_{3 / 2} \rightarrow 2 \mathrm{~s}{ }^{2} \mathrm{~S}_{1 / 2}\right)$ line of the solar corona was used as an example. It is formed by isotropic electron collisions and by partially anisotropic radiation coming from the same O VI line of the underlying transition region and then de-excited by spontaneous emission towards the ground state (resonance scattering). The O VI $1032 \AA$ line is linearly polarized, owing to the non spherical symmetry of its upper level $J=3 / 2$ and to the partial anisotropy of the incident radiation field, which can dominate the electron collisional excitation at the low densities which prevail at coronal altitudes. The other component of the fine-structure doublet $1037 \AA$ line is unpolarizable, due to the spherical symmetry of its upper and lower levels $J=1 / 2$. The two coronal 
re-emitted components' intensities can be dimmed by the Doppler effect, due to the outflow velocity field of the solar wind plasma (Rompolt 1967, 1969; Hyder \& Lites 1970; etc.).

This theory was recently used by Raouafi et al. (2002a, 2002b) when interpreting SUMER (the Solar Ultraviolet Measurements of Emitted Radiation: Wilhelm et al. 1995; Lemaire et al. 1997) SOHO (the Solar and Heliospheric Observatory; Domingo et al. 1995) their observations (Raouafi et al. 1999). This theory could also be used for interpreting observations by other instruments such as CDS (the Coronal Diagnostic Spectrometer: Harrison et al. 1995, 1997) and UVCS (the UltraViolet Coronagraph Spectrometer: Kohl et al. $1995,1997)$ on SOHO. In fact, attempts to measure polarization in coronal UV lines above the solar limb were also made by Thomas (2003) using the CDS normal-incidence spectrometer that is sensitive to polarization as is SUMER. His observations suggest that the emitted radiation of the cooler lines is dominated by resonance scattering beyond a certain distance over the limb, causing linear polarization. In addition, prospects in solar physics for the next few decades insist on the crucial importance of determining of vector magnetic and velocity fields for understanding of the heating and acceleration of the coronal plasma. Consequently, a number of future instruments that are currently being developed would include polarimeters designed to measure circular and linear polarization of UV lines: e.g. SUMI (Solar Ultraviolet Magnetograph Investigation: Porter et al. 2003), and LYOT (LYman Orbiter Telescope, solar highresolution imager - coronagraph mission, Vial et al. 2003). The development of these new instruments requires numerical simulations that predict the behavior and the order of magnitude of the sensitivity of the Stokes parameters to the velocity field.

To this end, we apply the analytical formulae that give the three first Stokes parameters of the re-emitted line obtained in $\mathrm{P}_{\mathrm{II}}$ and give quantitative numerical results. The sensitivity of the Stokes parameters and of the profile of the scattered line to the three components of the velocity vector are highlighted and discussed. The calculations are restricted to an emitting elementary volume around the plane of the sky. Integration along the line of sight is outside the scope of the present works, but see Raouafi \& Solanki (2004, 2006). In fact, polarization measurements of scattered lines which are optically thin most often concern observations made at the limb. For observations on the disk, the direct incoming radiation (not polarized) from the underlying layer of the solar atmosphere (i.e. transition region) is much more intense than the scattered coronal light under investigation.

We use the same notations as those of $\mathrm{P}_{\mathrm{II}}$, except when specified, and start from Eqs. (A1)-(A8) of $\mathrm{P}_{\mathrm{II}}$. For continuity, except when specified, we use the same atomic and solar data as those of Table 3 of Sahal-Bréchot et al. (1986), hereafter referred to as $\mathrm{P}_{\mathrm{I}}$ ). The limb-brightening coefficient is given by the analytical formula in $\mathrm{P}_{\mathrm{I}}$. We will also assume (as in $\mathrm{P}_{\mathrm{I}}$ ) that inhomogeneities of the incoming transition zone radiation are negligible. Therefore the incoming radiation intensity is assumed to have a cylindrical symmetry around the vertical, with a center-to-limb variation described by a limb-brightening coefficient (Eqs. (20) and (21) of $\mathrm{P}_{\mathrm{I}}$ ). Possible observations in

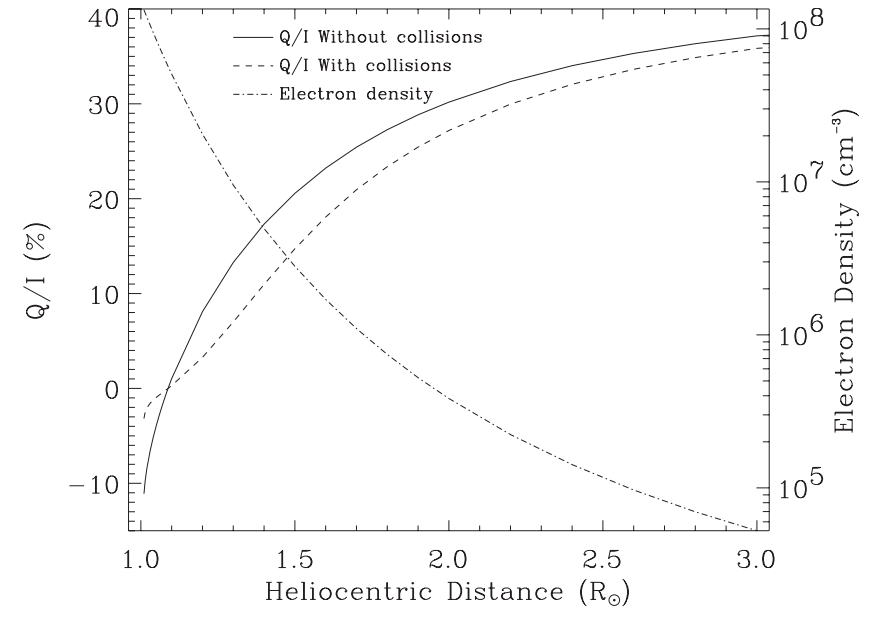

Fig. 1. $Q / I$ without velocity field, with and without collisions. The variation of the electronic density with heliocentric distance is taken from Doyle et al. (1999). If $Q / I$ is negative, the polarization direction is perpendicular to the limb and the fractional polarization is equal to $-Q / I$. If $Q / I$ is positive, the direction of polarization is parallel to the limb and the fractional polarization is equal to $Q / I$.

the vicinity of a coronal hole or of an active region are thus excluded from the present study.

The paper is organized as follows. In Sect. 2, we briefly recall the system used for calculation of the scattered line profiles and polarization parameters. In Sect. 3, we compare results obtained in our theory ( $\mathrm{P}_{\mathrm{II}}$, Doppler redistribution approach) to those obtained from the complete Doppler redistribution approximation. In Sect. 4, we apply the obtained theoretical results to the coronal O VI $1032 \AA$, then discuss and interpret the results. We give our conclusions in Sect. 5.

\section{Settings of the calculations}

As outlined in the introduction, the emitting elementary volume is considered above the solar limb in the plane of the sky. Thus the scattering angle $\theta$, which is the angle between the solar vertical (polar axis) and the line of sight is equal to $-\pi / 2$ (right angle scattering). This is illustrated in Fig. 3 of $\mathrm{P}_{\mathrm{II}}$.

In a first step, we calculate the polarization degree of the coronal O VI $1032 \AA$ A line with and without electron collisions. Electron densities taken into account are a function of the height over the solar limb. The velocity effect (Doppler dimming) and the magnetic field effect (Hanle effect, see Mitchell \& Zemansky 1934) are not taken into account. The variation of the electron density with the heliocentric distance is taken from Doyle et al. (1999). It is a combination of data recorded by SUMER (Doyle et al. 1999); UVCS (Kohl et al. 1999) and LASCO (Lamy et al. 1997). Figure 1 displays the obtained polarization rates and also the densities employed. The negative and positive values of the ratio $Q / I$ correspond to polarization vectors perpendicular and parallel to the solar limb, respectively. Figure 1 shows that the predicted polarization degree is noticeable, in particular at a height larger than $\sim 1.3 R_{\odot}$ from Sun center. Since the incident radiation field is cylindrically symmetric with respect to the polar axis and since the 

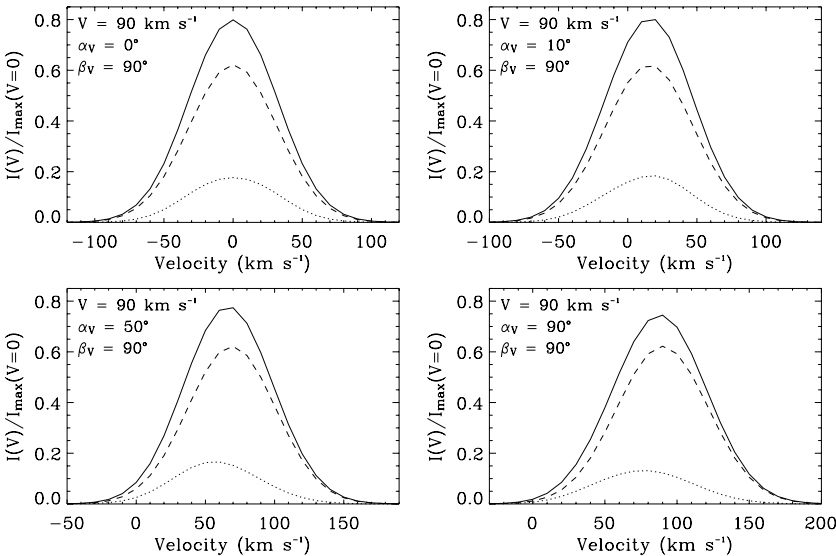

Fig. 2. Profiles of the O VI $1032 \AA$ line (solid lines) calculated for an outflow speed of $90 \mathrm{~km} \mathrm{~s}^{-1}$ and for different values of the polar angle $\alpha_{\mathrm{v}}$ and an azimuthal angle $\beta_{\mathrm{v}}$ equal to $90^{\circ}$ (outflow velocity field in the scattering plane). Ordonnae: ratio of the frequency $v$ and velocity field vector $\boldsymbol{V}$ dependence of the intensity $I(V, v)$ on the maximum value of the intensity in zero-velocity field. Abscissae: frequency in units of Doppler velocity shift $\left(\mathrm{km} \mathrm{s}^{-1}\right)$. These profiles correspond to a heliocentric distance of $1.05 R_{\odot}$. The dotted lines display the radiative contribution of the re-emitted line to the whole profile, and the dashed ones provide the collisional component (the electron density is equal to $\left.7 \times 10^{7} \mathrm{~cm}^{-3}\right)$. It is easy to note the evolution of the different parameters (width, shift and dimming) with the polar angle of the outflow velocity vector and the influence on the whole profile.

electron collisions are isotropic, the polarization direction of the re-emitted photons in the direction of the observer is parallel to the limb, except at very low altitudes $\left(h<1.1 R_{\odot}\right)$, where it becomes radial due to dominance of the limb-brightening effect. Indeed at low altitudes the polarization is masked by collisional excitations, which are predominant.

The relative importance of the contribution of collisions to the re-emitted line profiles is illustrated by Figs. 2 and 3 for two heliocentric distances of $1.05 R_{\odot}$ and $1.5 R_{\odot}$ and for the case of an outflow velocity field in the scattering plane.

By applying Eqs. (A1) to (A8) of $\mathrm{P}_{\mathrm{II}}$, we are now able to numerically study the effect of the velocity field on the Stokes parameters and on the spectral profile of the scattered coronal line. Equation (A1) predicts an asymmetry in the line profile which remains very small $(<1 \%)$ if we assume that the width $\Delta v_{D_{\mathrm{s}}}$ of the velocity distribution of the scattering ions (assumed to be Maxwellian in the atomic frame) is sufficiently larger than the width $\Delta v_{D_{i}}$ of the profile of OVI lines of the incident radiation coming from the solar transition region. In the opposite case, the asymmetry of the re-emitted line should be visible. This asymmetry will be neglected in the rest of this paper. The profile is, in fact, very close to a Gaussian shape; therefore, we will consider that the scattered line is symmetric and can be characterized by its Doppler width $w$, shift $d$, and dimming $D$.

It can also be shown that the frequency dependence of the Stokes $Q$ and $U$ parameters is the same as that of the intensity $I$. Therefore the scattered line can be also characterized by its polarization parameters: the global frequency integrated degree
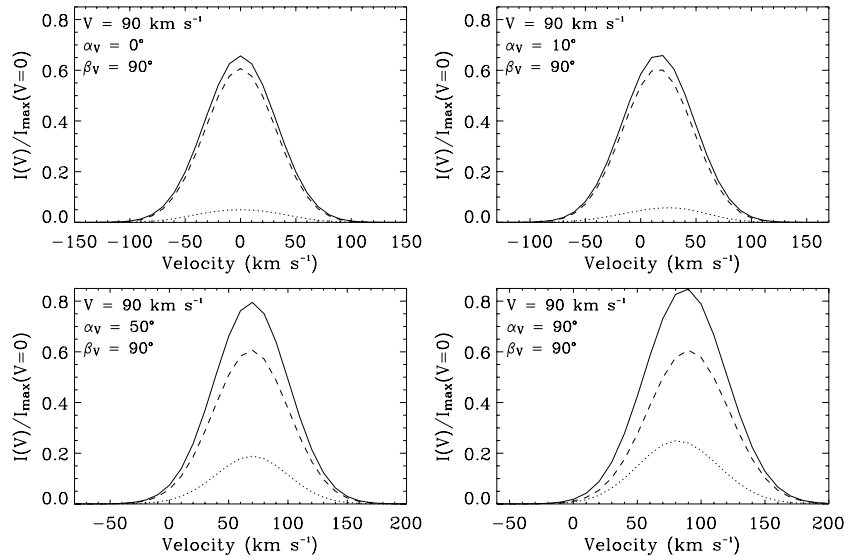

Fig. 3. The same as Fig. 2 but for a heliocentric distance of $1.5 R_{\odot}$. The electron density is equal to $1.5 \times 10^{7} \mathrm{~cm}^{-3}$.

of polarization $p$ and the direction of global polarization, $\phi$, relative to the tangent to the solar limb (the axis $(O x)$ or $(O X)$ in Figs. 2 and 3 of $\mathrm{P}_{\mathrm{II}}$ ).

We define the dimming and the polarization parameters of the scattered line as follows:

$D=I_{\max }(\boldsymbol{V}) / I_{\max }(\boldsymbol{V}=0) ;$

$p=\frac{\sqrt{Q^{2}+U^{2}}}{I}$

$\cos (2 \phi)=\frac{Q}{\sqrt{Q^{2}+U^{2}}} ;$

$\sin (2 \phi)=\frac{U}{\sqrt{Q^{2}+U^{2}}}$,

where $w$ is the $(1 / e)$ width that is obtained by applying a Gaussian fit to the scattered line profile. It is related to the full width at half-maximum, $F W H M$, by

$F W H M=2 \sqrt{\log 2} w$.

The direction of polarization $\phi$ is defined between 0 and $\pi$.

\section{Comparison with the complete redistribution approximation}

Since the scattering is monochromatic in the atomic rest frame, the theory developed in $\mathrm{P}_{\mathrm{II}}$ is an approximation of Doppler redistribution. It is denoted as "velocity-coherence" approximation by Landi Degl'Innocenti \& Landolfi (2004), and corresponds to case I of redistribution process in the formalism developed by Hummer (1962) and discussed in the book by Mihalas (1978, p. 412). We compare it to the complete redistribution in velocities approximation (cf. Sahal-Bréchot \& Choucq-Bruston 1994). A comprehensive discussion of the validity conditions of the "velocity-coherence" approximation and of the complete redistribution in velocities approximation has been made by Landi Degl'Innocenti \& Landolfi (2004, Sect. 2 of Chap. 13). Also see Oxenius (1986). 
Physically, the complete redistribution in velocities means that the velocity is completely randomly redistributed in the comoving frame during the re-emission process, i.e. when the ion is in its excited state. Then the distribution of the velocities of the atom (ion) in the excited state is the same as that of the ground level, the Maxwell distribution in the comoving frame. On the contrary, the coherent redistribution in velocities (the one of the present paper) means that the atomic (ionic) velocity is unchanged during the re-emission process. The velocitycoherence approximation is valid, if during the lifetime of the atomic (ionic) state, no ion-ion elastic collisions take place that give rise to velocity changes of the atom (ion). By contrast, the complete redistribution in velocities is valid if elastic collisions with the excited atom (ion) occur that change its velocity. In practice (see Landi Degl'Innocenti \& Landolfi 2004), in outer layers of stellar atmospheres the densities are so weak that these elastic collisions are negligible and the "velocitycoherence" approximation is valid. However, the complete redistribution approximation in velocities is most often used.

For simplicity, we ignore the polarization of the re-emitted spectral line for the moment. Besides, this does not alter the generality of the reasoning. Therefore only the first Stokes parameter $I(v)$ enters the present discussion. In our theory (the so-called Mihalas "Case I"), we can write (cf. $\left.\mathrm{P}_{\mathrm{II}}\right)$ :

$$
\begin{aligned}
I(v) \mathrm{d} v & =\varepsilon(v) \mathrm{d} v \\
& =\frac{1}{4 \pi} \mathrm{d} v_{\mathrm{A}_{Z}} \iint N_{\mathrm{A}_{u}}\left(\boldsymbol{v}_{\mathrm{A}}\right) F\left(\boldsymbol{v}_{\mathrm{A}}\right) \mathrm{d} v_{\mathrm{A}_{X}} \mathrm{~d} v_{\mathrm{A}_{Y}}
\end{aligned}
$$

with $\varepsilon(v)$ the emissivity coefficient of the line emitted between the excited level $u$ and the ground one $l$ at frequency $v_{0}=v_{u l}$. $N_{\mathrm{A}_{u}}\left(\boldsymbol{v}_{\mathrm{A}}\right)$ is the population of the atomic level $u$ (of the OVI in the present study) with the velocity vector $\boldsymbol{v}_{\mathrm{A}} . F\left(\boldsymbol{v}_{\mathrm{A}}\right)$ is the atomic distribution of velocities (normalized to unity), and $v_{\mathrm{A}_{Z}}$ the component of $\boldsymbol{v}_{\mathrm{A}}$ along the line of sight. It is related to the frequency of the re-emitted photons by $v=v_{0}\left(1+v_{\mathrm{A}_{z}} / c\right)$. The intensity of the scattered line is given in number of photons per unit of solid angle, per unit of time, per unit of frequency, and per unit of volume.

Since the internal (atomic levels) and external (velocities) atomic variables are decoupled (Sect. 2.2 of $\mathrm{P}_{\mathrm{II}}$ ), the total probability of having an atom in the excited state $u$ with velocity $v_{\mathrm{A}}$ is equal to $N_{\mathrm{A}_{u}}\left(\boldsymbol{v}_{\mathrm{A}}\right) F\left(\boldsymbol{v}_{\mathrm{A}}\right)$. Following Oxenius (1986), we can define the population $N_{\mathrm{A}_{u}}$ integrated over all velocities of the level $u$ by

$$
N_{\mathrm{A}_{u}}=\int N_{\mathrm{A}_{u}}\left(\boldsymbol{v}_{\mathrm{A}}\right) F\left(\boldsymbol{v}_{\mathrm{A}}\right) \mathrm{d}^{3} \boldsymbol{v}_{\mathrm{A}},
$$

and consequently we must define a distribution of velocities for each internal level $u$

$$
F_{u}\left(\boldsymbol{v}_{\mathrm{A}}\right)=\frac{N_{\mathrm{A}_{u}}\left(\boldsymbol{v}_{\mathrm{A}}\right) F\left(\boldsymbol{v}_{\mathrm{A}}\right)}{N_{\mathrm{A}_{u}}} .
$$

Equation (3) can then be written as

$$
\begin{aligned}
I(v) \mathrm{d} v & =\varepsilon(v) \mathrm{d} v \\
& =\frac{1}{4 \pi} \mathrm{d} v_{\mathrm{A}_{Z}} \iint N_{\mathrm{A}_{u}} F_{u}\left(\boldsymbol{v}_{\mathrm{A}}\right) \mathrm{d} v_{\mathrm{A}_{X}} \mathrm{~d} v_{\mathrm{A}_{Y}} .
\end{aligned}
$$

The complete Doppler redistribution approximation consists in assuming that the velocity distributions of all the internal levels $u$ are the same (Oxenius 1986, p.169), i.e.: $F_{u}\left(\boldsymbol{v}_{\mathrm{A}}\right)=F\left(\boldsymbol{v}_{\mathrm{A}}\right)$.

In fact, the atomic profiles are equal since they are assumed to be Dirac $\delta$-functions (see $\mathrm{P}_{\mathrm{II}}$ ). Thus all the above is the same thing as saying that the emission profile $\psi_{v}$ is equal to the absorption profile $\phi_{v}$ (Mihalas 1978; Oxenius 1986), which is the complete redistribution approximation. Therefore, in the complete redistribution approximation in velocities, the first Stokes parameter (emissivity coefficient $\varepsilon_{\mathrm{cr}}$ at the frequency $v$ ) is equal to

$$
I_{\mathrm{cr}}(v) \mathrm{d} v=\varepsilon_{\mathrm{cr}}(v) \mathrm{d} v=\frac{1}{4 \pi} N_{\mathrm{A}_{u}} F_{Z}\left(v_{\mathrm{A}_{Z}}\right) \mathrm{d} v_{\mathrm{A}_{Z}}
$$

where $F_{Z}\left(v_{\mathrm{A}_{Z}}\right)$ is the $Z$ component of the distribution of velocities in the laboratory frame (cf. $\mathrm{P}_{\mathrm{II}}$ ).

We then extend the same reasoning to the other Stokes parameters. As pointed out by Landi Degl'Innocenti \& Landolfi (2004), when the complete redistribution approximation on velocities is fully justified, polarization phenomena turns out, in general, to be of marginal importance, because densities are not weak and thus depolarizing collisions that redistribute the internal (Zeeman) states of the atom (ion) are also important. The complete redistribution approximation in velocities is most often used in radiative transfer for polarized radiation, especially for the solution of non-LTE problems. In fact, that approach, though incorrect in principle, is adopted to get an approximate solution to a problem that would be insoluble in practice.

We now obtain the expression of the three Stokes parameters of interest in the complete Doppler redistribution approximation. First we integrate the atomic density-matrix elements that were obtained for the velocity $\boldsymbol{v}_{\mathrm{A}}$ (formula (30) of $\mathrm{P}_{\mathrm{II}}$ ) over all atomic velocities. Secondly we obtain the Stokes profiles in the complete redistribution approximation by multiplying the precedent ones by $F_{Z}\left(v_{\mathrm{A}_{Z}}\right)$. We have then

$$
\begin{aligned}
\left(\begin{array}{l}
I_{\mathrm{cr}}(v) \\
Q_{\mathrm{cr}}(v) \\
U_{\mathrm{cr}}(v)
\end{array}\right) \mathrm{d} v= & \frac{1}{4 \pi} F_{Z}\left(v_{\mathrm{A}_{Z}}\right) \\
& \times\left[\iiint\left(\begin{array}{c}
I\left(\boldsymbol{v}_{\mathrm{A}}\right) \\
Q\left(\boldsymbol{v}_{\mathrm{A}}\right) \\
U\left(\boldsymbol{v}_{\mathrm{A}}\right)
\end{array}\right) F\left(\boldsymbol{v}_{\mathrm{A}}\right) \mathrm{d}^{3} v_{\mathrm{A}}\right] \mathrm{d} v_{\mathrm{A}_{Z}}
\end{aligned}
$$

where $I, Q$ and $U$ are the Stokes parameters in the Doppler redistribution of $\mathrm{P}_{\mathrm{II}}$.

Next, as in $\mathrm{P}_{\mathrm{II}}$, we assume a Maxwellian velocity distribution for the scattering ions with a drift $\boldsymbol{V}$ :

$$
F\left(\boldsymbol{v}_{\mathrm{A}}\right)=\left(\frac{m_{\mathrm{A}}}{2 \pi k T}\right)^{3 / 2} \exp \left(-\frac{m_{\mathrm{A}}}{2 k T}\left|\boldsymbol{v}_{\mathrm{A}}-\boldsymbol{V}\right|^{2}\right)
$$

where $c$ is the speed of light, $k$ the Boltzmann constant, and $T$ and $m_{\mathrm{A}}$ are the atomic temperature and mass, respectively. The distribution of velocities is Maxwellian in all directions in the atomic frame (at rest), so we have for the $v_{\mathrm{A}_{Z}}$ component

$F_{Z}\left(v_{\mathrm{A}_{Z}}\right)=\left(\frac{m_{\mathrm{A}}}{2 \pi k T}\right)^{1 / 2} \exp \left[-\frac{m_{\mathrm{A}}}{2 k T}\left(v_{\mathrm{A}_{Z}}-V_{Z}\right)^{2}\right]$. 
Therefore the formulae (A1) to (A7) of Appendix A of $\mathrm{P}_{\mathrm{II}}$ (case of the coronal O VI $1032 \AA$ line) become in the complete redistribution approximation:

$$
\begin{aligned}
\left(\begin{array}{l}
I_{\mathrm{cr}}(v) \\
Q_{\mathrm{cr}}(v) \\
U_{\mathrm{cr}}(v)
\end{array}\right) \mathrm{d} v= & \mathrm{d} v \frac{1}{\sqrt{\pi} \Delta v_{D_{\mathrm{s}}}} \\
& \times \exp \left[-\left(\frac{v-v_{0}-\Delta v_{Z}}{\Delta v_{D_{\mathrm{s}}}}\right)^{2}\right]\left(\begin{array}{c}
I \\
Q \\
U
\end{array}\right)
\end{aligned}
$$

where $\Delta v_{D_{\mathrm{s}}}=\frac{v_{0}}{c} \sqrt{\frac{2 k T}{m_{\mathrm{A}}}}$, and where $I, Q, U$ are the $v$-integrated Stokes parameters of Eq. (A8) of $\mathrm{P}_{\mathrm{II}}$.

Therefore, in the complete redistribution approximation, the Doppler width $w_{\text {cr }}$ of the radiative component of the reemitted line is equal to $\Delta v_{D_{\mathrm{s}}}$ and does not depend on the macroscopic velocity vector $\boldsymbol{V}$ of the scattering ions. The Doppler shift in the complete redistribution approximation is equal to $d_{\mathrm{cr}}=v_{0} V_{Z} / c=\Delta v_{Z}$, and thus is a function only of the lineof-sight component $V_{Z}$ of $\boldsymbol{V}$. Consequently, the profile of the radiative component is the same as the one for the collisional component. The only difference lies in the amplitude that determines the contribution of each of them to the whole line profile. In addition the frequency-integrated Stokes parameters, and consequently the degree and direction of polarization, are the same in both the Doppler redistribution theory and the complete redistribution approximation. The differences concern only the Doppler shift and width.

In order to compare the results of both the Doppler redistribution theory and the complete redistribution approximation and then to study the sensitivity of those physical quantities of interest for the velocity field vector, we calculate the ratios $w(\boldsymbol{V}) / w_{\text {cr }}$ and $d(\boldsymbol{V}) / d_{\text {cr }}$ (see Sect. 4). In addition, these quantities converge to unity at very large distances from Sun center when the incident radiation can be considered as purely directive. This is due to the fact that the Doppler width and shift in the Doppler redistribution theory converge towards $\Delta v_{D_{\mathrm{s}}}$ and $\Delta v_{Z}$, respectively (see Sahal-Bréchot et al. 1992 and $\mathrm{P}_{\mathrm{II}}$ ).

\section{Numerical results: Application to the O VI $1032 \AA$ coronal line}

We apply the theory developed in $\mathrm{P}_{\text {II }}$ to calculate the polarization parameters and the Doppler shift $d$, width $w$, and intensity dimming $D$ of the O VI $1032 \AA$ line emitted in the solar corona. The profile of the incident solar disk line is assumed to be Gaussian with a width equal to $30 \mathrm{~km} \mathrm{~s}^{-1}$. The profile of the scattered line is also assumed to be Gaussian with a width $\Delta v_{D_{\mathrm{s}}}$ equal to $55 \mathrm{~km} \mathrm{~s}^{-1}$.

The calculations are then achieved for four coronal altitudes: $1.05 R_{\odot}$ from Sun center (very close to the solar limb, where the contribution of the limb-brightening to the radiative component is very important): $1.5 R_{\odot} ; 2.0 R_{\odot}$; and 5.0 $R_{\odot}$, where the incident radiation becomes almost purely directive. The electron density is given in Fig. 1 as a function of the heliocentric distance. The velocity distribution is assumed to be a Maxwellian with a drift velocity vector $\boldsymbol{V}$ that can be equated to the outflow speed of the solar wind. The outflow velocity field vector $\boldsymbol{V}$ is defined by its strength $V$, by the polar angle $\alpha_{\mathrm{v}}$ made with the vertical to the solar surface, and by the azimuth angle $\beta_{\mathrm{v}}$ made with the tangent to the solar limb (see Fig. 3 in $\mathrm{P}_{\mathrm{II}}$ ). The results are given for $\left(0 \leq \alpha_{\mathrm{v}} \leq \pi / 2\right)$ and $\left(0 \leq \beta_{\mathrm{v}} \leq \pi / 2\right)$. The results for $\left(\pi / 2<\alpha_{\mathrm{v}}<2 \pi\right)$ and $\left(\pi / 2<\beta_{\mathrm{v}}<2 \pi\right)$ can be deduced from the present ones by symmetry (cf. Sect. 3.2 and Fig. 4 of $\mathrm{P}_{\mathrm{II}}$ ).

\subsection{Polarization parameters}

As in Hanle studies, we display the velocity dependence of the fractional polarization (or degree of linear polarization) $p(\boldsymbol{V})$ and the direction of linear polarization and compare them to those obtained in a zero-velocity field. Figures 4-7 display the abaques of linear polarization of the O VI $1032 \AA$ coronal line. The top and bottom-left panels of each figure display the relative fractional polarization $p(\boldsymbol{V}) / p(\boldsymbol{V}=0)$ as a function of the rotation angle $\phi$ of the polarization direction with respect to the parallel to the solar limb for different values of the macroscopic velocity vector $\boldsymbol{V}$ and for the four chosen heliocentric distances. The bottom-right panels of the same figures display the relative fractional polarization as a function of the azimuthal angle $\beta_{\mathrm{v}}$ of the velocity field. In this case the plane of polarization is parallel to the solar limb (cf. below).

On the one hand, the velocity field modifies both the polarization degree and the direction of polarization, while on the other hand, the collisions only reduce the polarization degree. In fact, the direction of polarization is not affected by collisions, because $Q$ and $U$ contain only those anisotropy effects of the incident radiation that are not sensitive to collisions. At low altitudes the collisional component is important, thus the polarization degree is considerably reduced by collisions (cf. Fig. 1 for the zero-velocity field case). For giving an order of magnitude at $1.5 R_{\odot}$ from Sun center, excitations by collisions are roughly two times greater than those by radiation. At $2 R_{\odot}$, radiative excitations are ten times greater than excitations by collisions. The polarization degree is more sensitive to the velocity field at low altitudes than at high altitudes (compare Figs. 4 and 7). This is due to the fact that collisions are dominant at low altitudes. The intensity $I$ is hardly sensitive to the incident radiation, thus to the velocity field, and the polarization degree given by the ratio $p=\sqrt{Q^{2}+U^{2}} / I$ becomes significantly sensitive to the velocity field. On the contrary, at high altitudes, collisions can be neglected because radiative excitations are predominant. Thus, without collisions (the case at high altitudes), the sensitivity of the three Stokes parameters $I$, $Q$, and $U$ to the velocity field is almost the same, and the ratio giving the polarization degree becomes nearly insensitive to the velocity field.

The top-left panels of Figs. 4-7 correspond to a vertical velocity field, i.e. with the same direction as the symmetry axis of the incident radiation field. Owing to the Doppler dimming effect, the scattering atoms absorb less radiation since the absorption takes place in the wings of the incident line. At very low altitudes most of the radiation comes from the limb of the spherical cap, hence the anisotropy increases when the velocity increases and the polarization degree increases. The drift has a 

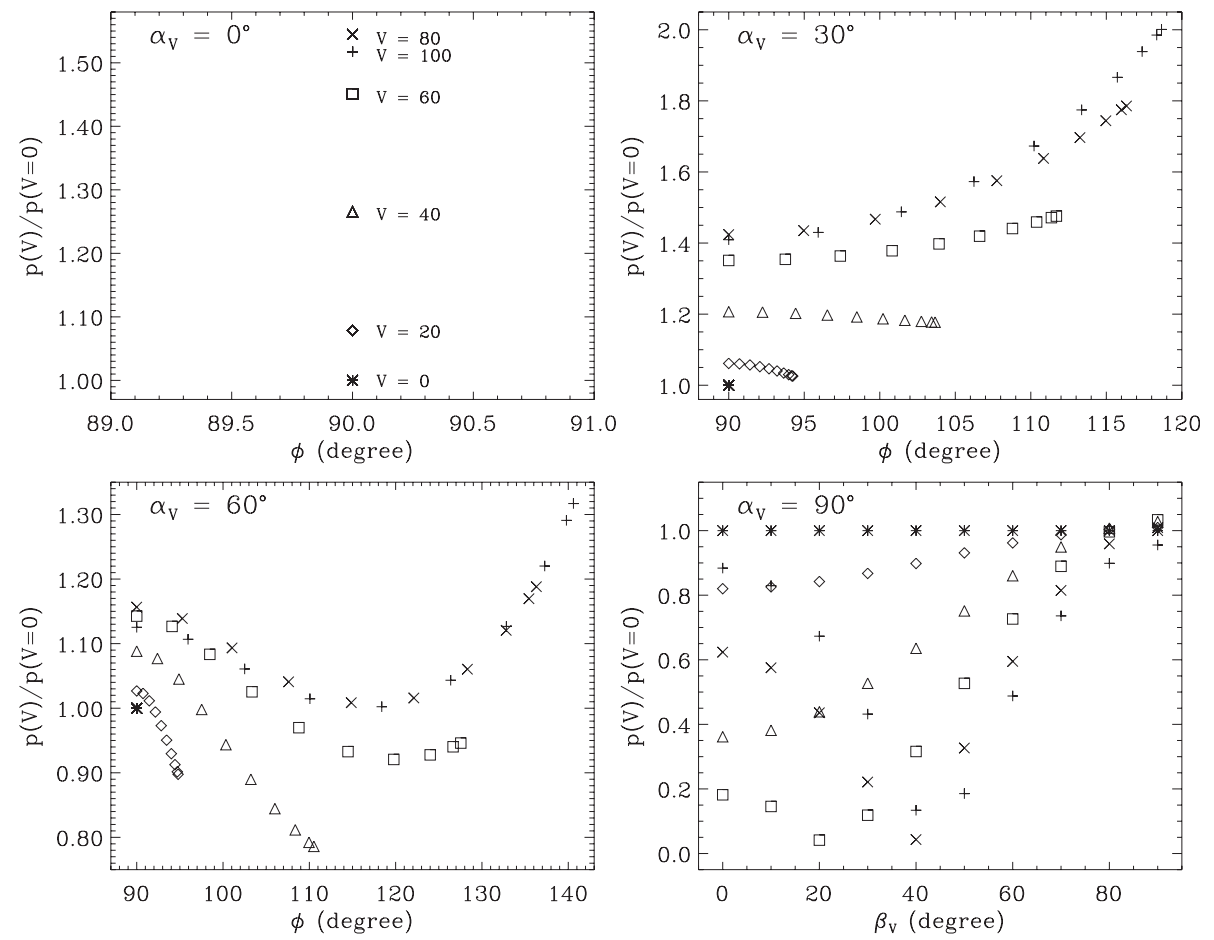

Fig. 4. Effect of the outflow velocity field vector on the polarization parameters of the O VI $1032 \AA$ scattered line for an altitude of $1.05 R_{\odot}$ from Sun center. The electron density is equal to $7 \times 10^{7} \mathrm{~cm}^{-3}$. In a zero-velocity field, the degree of polarization $p(V=0)=0.0095$, and the direction of polarization is perpendicular to the solar limb (owing to the limb brightening effect that is very important at very low altitudes). The different velocity vectors are described by their outflow speeds $\left(0-100 \mathrm{~km} \mathrm{~s}^{-1}\right)$ and their directions are given by $\alpha_{\mathrm{v}}\left(0,30,60,90^{\circ}\right)$, and $\beta_{\mathrm{v}}$ which are ranging from $0^{\circ}$ to $90^{\circ}$ per steps of $10^{\circ}$. The different symbols are related to the speed values (in $\mathrm{km} \mathrm{s}^{-1}$ ) according to the top-left panel. Top and bottom-left panels $\left(\alpha_{\mathrm{v}}=0,30,60^{\circ}\right)$ display the variation of the linear polarization degree versus the rotation angle of the polarization direction (with respect to the tangent to solar limb that is the reference axis) of the scattered line. For $\beta_{\mathrm{v}}=90^{\circ}$ the polarization vector is always perpendicular to the solar surface $\left(\phi=90^{\circ}\right)$. For $\alpha_{\mathrm{v}}=90^{\circ}$ (bottom-right panel), the direction of polarization is perpendicular to the solar surface for every value of $\beta_{\mathrm{v}}$, thus the variation of the degree of linear polarization as a function of $\beta_{\mathrm{v}}$ is plotted in this case.

polarizing effect. However, for high altitudes, the limb brightening is less important; most of the radiation comes along the vertical, thus the anisotropy decreases when the velocity increases and the polarization degree decreases with the velocity. The drift has a depolarizing effect. The direction of polarization is not modified because the cylindrical symmetry around the vertical is conserved.

For inclined outflows (with respect to the vertical), the cylindrical symmetry is broken and coherences appear in the polarization matrix of the incident radiation. Therefore the degree of polarization is modified and the plane of polarization rotated, as shown by the other diagrams.

The bottom-right panels of Figs. 4-7 $\left(\alpha_{\mathrm{v}}=90^{\circ}\right)$ correspond to a horizontal velocity field. The polarization direction is unchanged and the plane of polarization is parallel to the solar limb. In this case the contributions to $U$ of the incident radiation coming from half-spaces $\left(0 \leq \beta_{\mathrm{v}} \leq \pi\right)$ and $\left(\pi \leq \beta_{\mathrm{v}} \leq 2 \pi\right)$ are opposite and cancel each other.

For the other polar angles (top-right and bottom-left panels) the situation is more complex. A particular case concerns a velocity field in the scattering plane $\left(\beta_{\mathrm{v}}=90^{\circ}\right)$. In that case $U=0$, because the contributions to $U$ coming from above $\left(-\pi / 2 \leq \beta_{\mathrm{v}} \leq \pi / 2\right)$ and below $\left(\pi / 2 \leq \beta_{\mathrm{v}} \leq 3 \pi / 2\right)$ the scattering plane are opposite, thereby cancelling each other out. So the rotation $\phi$ of the direction of polarization is zero.
For $\beta_{\mathrm{v}}=0^{\circ}$ the rotation of polarization is maximum and this maximum depends on the polar angle $\alpha_{\mathrm{v}}$ and on the velocity strength $V$.

The directions $\left(\alpha_{\mathrm{v}}=90^{\circ}, \beta_{\mathrm{v}}=0^{\circ}\right)$ and $\left(\alpha_{\mathrm{v}}=90^{\circ}\right.$, $\beta_{\mathrm{v}}=90^{\circ}$ ) are parallel to the limb and along the line of sight, respectively. The direction $\alpha_{\mathrm{v}}=0^{\circ}$ is vertical. Hence for outflow velocity vectors of the scattering atoms parallel to one of the three principal axes (solar vertical, tangent to the limb, and the line of sight), the rotation of the plane of polarization is zero.

As for $1.05 R_{\odot}$ (Fig. 4), at low altitudes and in zero outflow speed, the direction of polarization is perpendicular to the solar surface, because the center-to-limb brightening effect is predominant. The radiative component results mostly from the radiation coming from the limb of the spherical cap in addition to electron collisions. Generally the degree of polarization $p(\boldsymbol{V})$ of the scattered radiation increases with outflow speed $\boldsymbol{V}$ and with azimuth angle $\beta_{\mathrm{v}}$ for low values of the polar angle $\alpha_{\mathrm{v}}$ (see top-right panel of Fig. 4), except for low values of $V$ where the degree of polarization decreases slightly with $\beta_{\mathrm{v}}$. In fact, when the velocity drift $V$ increases, the anisotropy increases, because the incident radiation comes more and more from the limb of the spherical cap, so that the polarization degree increases. For higher values of the polar angle, variation of the degree of polarization is more complex. The degree of 

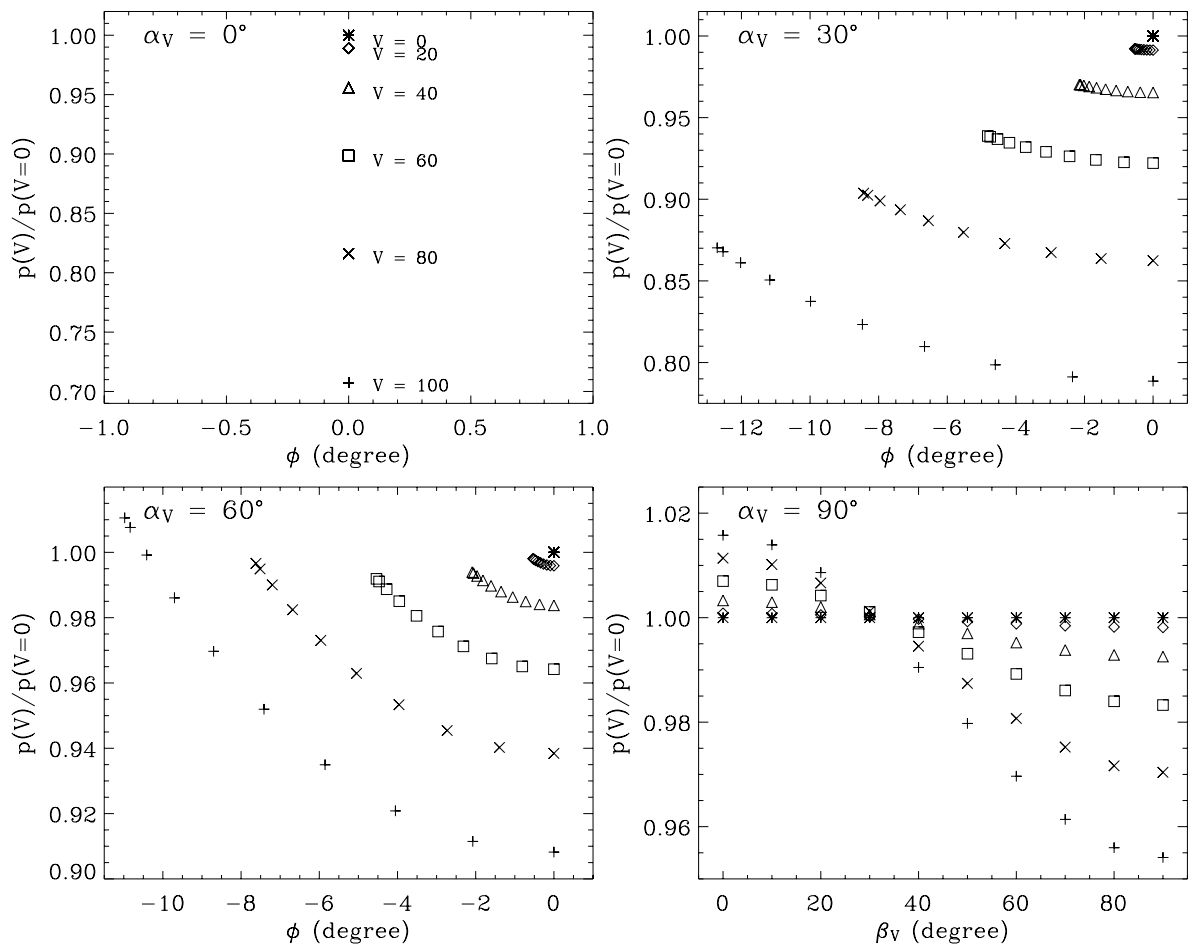

Fig. 5. Same plots as those of Fig. 4 but for $1.5 R_{\odot}$ from Sun center. The electron density is equal to $1.5 \times 10^{7} \mathrm{~cm}^{-3}$. At $V=0$ the fractional polarization is equal to 0.147 and the direction of polarization is parallel to the limb (cf. Fig. 2). Notice that for $\beta_{\mathrm{v}}=90^{\circ}$ the polarization vector is always parallel to the solar surface $\left(\phi=0^{\circ}\right)$. For $\alpha_{\mathrm{v}}=90^{\circ}$ (bottom-right panel), the direction of polarization is parallel to the solar surface for every value of $\beta_{\mathrm{v}}$, thus the variation of the degree of linear polarization as a function of $\beta_{\mathrm{v}}$ is plotted in this case as in Fig. 4.
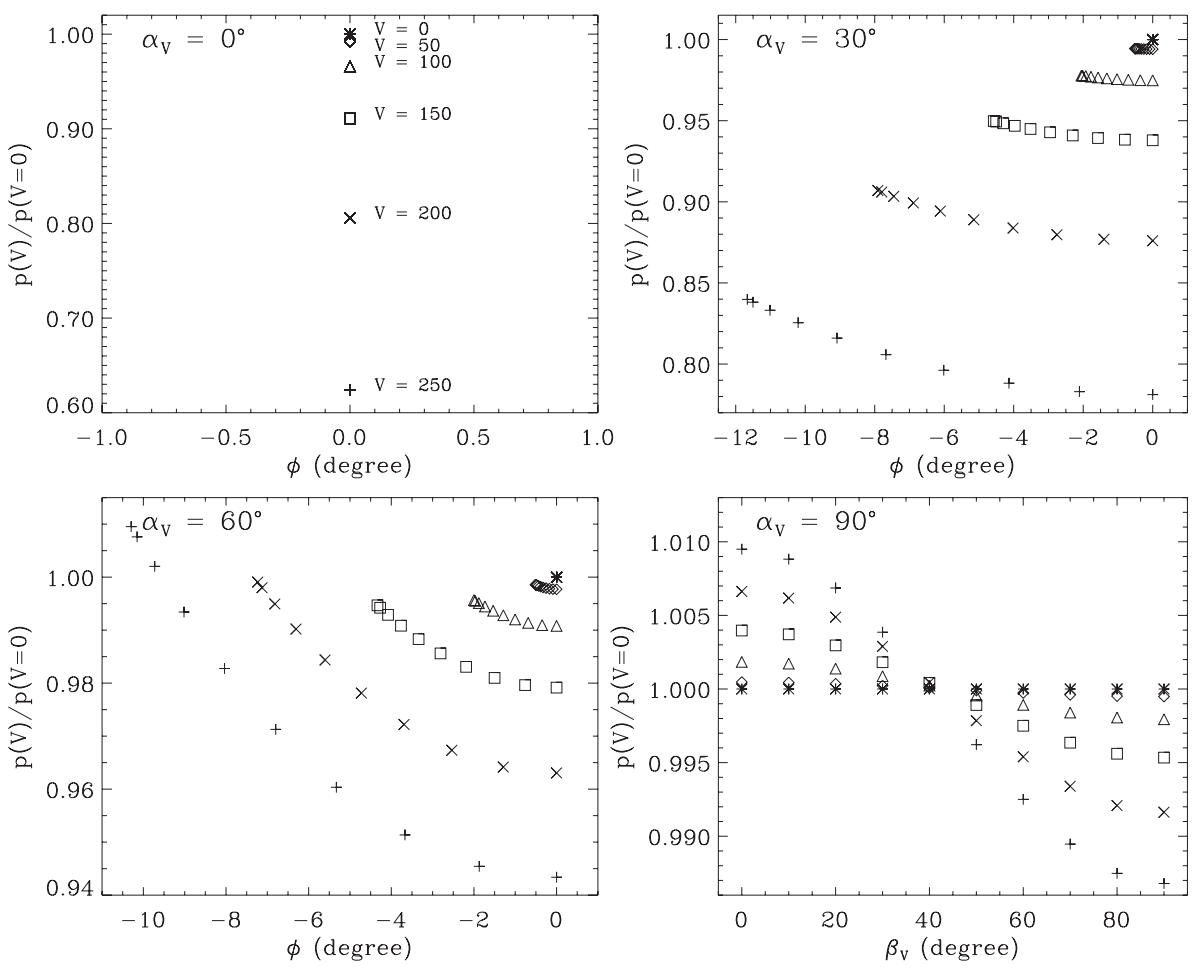

Fig. 6. Same plots as those of Fig. 4 but for $2.0 R_{\odot}$ from Sun center. The electron density is equal to $3.8 \times 10^{5} \mathrm{~cm}^{-3}$. At $V=0$ the fractional polarization is equal to 0.272 and the direction of polarization is parallel to the limb.

polarization decreases first and then increases beyond a given value of $\beta_{\mathrm{v}}$, characteristic of each value of $\alpha_{\mathrm{v}}$.

For medium distances $\left(1.5\right.$ and $\left.2 R_{\odot}\right)$, the degree of polarization of the re-emitted radiation generally decreases as the outflow speed of the emitting ions increases. It increases with the polar angle $\alpha_{\mathrm{v}}$ and with the azimuth angle $\beta_{\mathrm{v}}$. The decrease in $p(\boldsymbol{V})$ with the increase in the outflow speed is due to the Doppler dimming effect, which is roughly proportional to the component along the solar vertical of the velocity drift $\boldsymbol{V}$ of the scattering ions. On the one hand, the amount of the absorbed radiation and then the re-emitted one decreases when the velocity drift of the scattering ions is higher, and thus the contribution of the non-polarizing collisional component becomes more important. On the other hand, when $\alpha_{\mathrm{v}}$ increases, the component of the velocity drift along the solar vertical decreases, which implies an increase in the degree of linear polarization of the re-emitted radiation.

At high altitudes above the solar limb $\left(5 R_{\odot}\right.$ : Fig. 7$)$, the incident radiation beam can be considered as almost purely directive. The polarization vector remains in a first order 

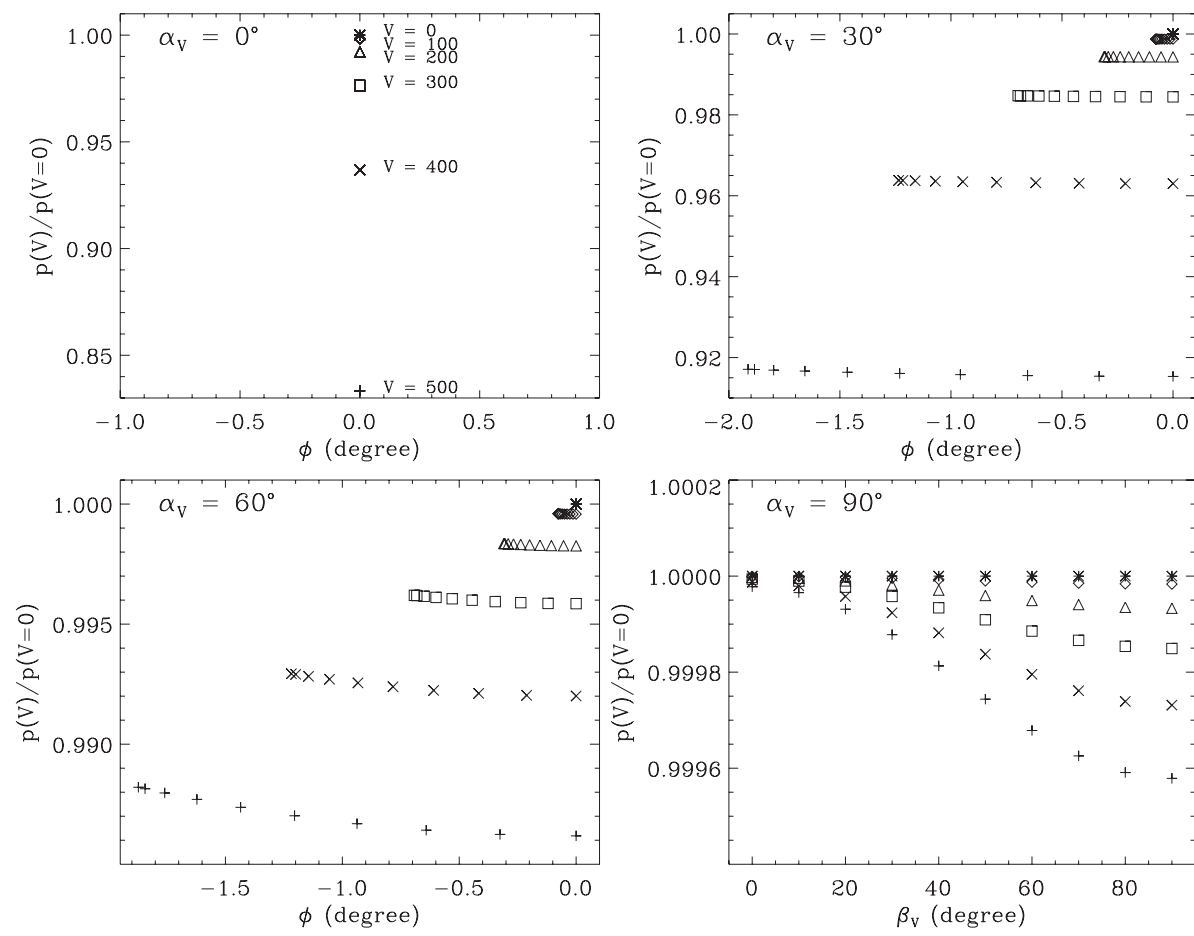

Fig. 7. Same plots as those of Fig. 4 but for $5.0 R_{\odot}$ from Sun center. The electron density is equal to $1.1 \times 10^{4} \mathrm{~cm}^{-3}$. At $V=0$ the fractional polarization is equal to 0.4 and the direction of polarization is parallel to the limb. The polarization vector is nearly parallel to the solar limb due to the fact that the incident radiation is almost a pure directive beam. approximation parallel to the solar limb, i.e. small rotation angle of the direction of polarization, top, and bottom-left panels of the same figure. The variation of the polarization degree with the outflow speed $V, \alpha_{\mathrm{v}}$, and $\beta_{\mathrm{v}}$ is similar to the previous case.

For the case of non-right angle scattering, Raouafi et al. (2002a) made a detailed study of the variation of the polarization parameters of the O VI $1032 \AA$. The effect of an eventual anisotropy in the velocity distribution of the scattering ions in the atomic frame has been studied by Raouafi \& Solanki (2003).

\subsection{The intensity profile: Doppler width, shift, and dimming}

Figures 8-11 display the variation of the ratios ("Doppler redistribution/complete redistribution" quantities) of the Doppler widths $\left(w(\boldsymbol{V}) / w_{\mathrm{cr}}\right.$, top panels), the Doppler shifts $\left(d(\boldsymbol{V}) / d_{\mathrm{cr}}\right.$, middle panels), and the dimming $\left(D=I_{\max }(\boldsymbol{V}) / I_{\max }(\boldsymbol{V}=0)\right.$, bottom panels) as a function of the polar angle $\alpha_{\mathrm{v}}$ and for different values both of the azimuth angle $\beta_{\mathrm{v}}$ and of the macroscopic outflow speed $V$ of the scattering ions. Computations of the re-emitted profile are achieved for the same altitudes as in the previous section. Figures 8-11 correspond to heliocentric distances equal to $1.05,1.5,2.0$, and $5.0 R_{\odot}$, respectively.

The obtained results are first briefly described and then we interpret the variation of the different spectroscopic parameters as a function of the velocity vector of the scattering ions.

\subsubsection{Case of low altitudes: $r=1.05 R_{\odot}$}

For weak and medium values of the outflow speeds $(V=$ $30-60 \mathrm{~km} \mathrm{~s}^{-1}$, respectively), the relative width $w(\boldsymbol{V}) / w_{\mathrm{cr}}$ of the re-emitted line profile (Fig. 8, top panels) decreases with the polar and azimuth angles, $\alpha_{\mathrm{v}}$ and $\beta_{\mathrm{v}}$, respectively, for low values of $\beta_{\mathrm{v}}$. For values of $\beta_{\mathrm{v}}$ higher than $\sim 50^{\circ}$ for $V=$ $30 \mathrm{~km} \mathrm{~s}^{-1}$ and $\sim 10^{\circ}$ for $V=60 \mathrm{~km} \mathrm{~s}^{-1}$, the width ratio decreases first until a given limit of $\alpha_{\mathrm{v}}$, beyond which it increases. However, for high values of $V\left(V=90 \mathrm{~km} \mathrm{~s}^{-1}\right)$ (top-right panel of Fig. 8), the variation of the width ratio is similar to the preceding case for low values of the azimuth angle and more complex for higher values of $\beta_{\mathrm{v}}$.

For weak outflow velocities, the shift ratio $d(\boldsymbol{V}) / d_{\text {cr }}$ increases with the azimuth angle for a given value of the polar angle. The shift ratio decreases with the polar angle for values of $\beta_{\mathrm{v}} \leq 50^{\circ}$ and increases for higher values of the azimuth angle (see medium-right panel of Fig. 8). For medium and high outflow speed (see Fig. 8 for $V=60$ and $90 \mathrm{~km} \mathrm{~s}^{-1}$ ), the shift ratio is decreasing with the polar angle and varies more slowly with the azimuth angle for velocity vectors having a small inclination with respect to the solar vertical. However, for higher values of $\alpha_{\mathrm{v}}$, the shift increases with both angles.

The dimming $D$ (bottom panels of Fig. 8) generally decreases with both polar and azimuth angles except for high outflow speeds where an increase with $\alpha_{\mathrm{v}}$ and also with $\beta_{\mathrm{v}}$ is remarkable.

That can be explained as follows: the re-emitted line profile is the result of two contributions, a collisional profile and a radiative one. The width of the collisional profile is equal to $\Delta v_{D_{\mathrm{s}}}$ and its shift is equal to $\Delta v_{Z}$ in both Doppler and complete redistribution approximation (see dashed line in Fig. 2). The collisional profile is not sensitive to the Doppler dimming that only affects the radiative profile. Thus, the width and amplitude of the collisional profile do not depend on the outflow speed of the reemitting ions. Only the Doppler shift can change, depending on the direction of the velocity drift of the scattering ions. The line width of the radiative profile is sensitive to the Doppler dimming effect and is slightly smaller than that of the collisional one (dotted line in Fig. 2). This is why for small 

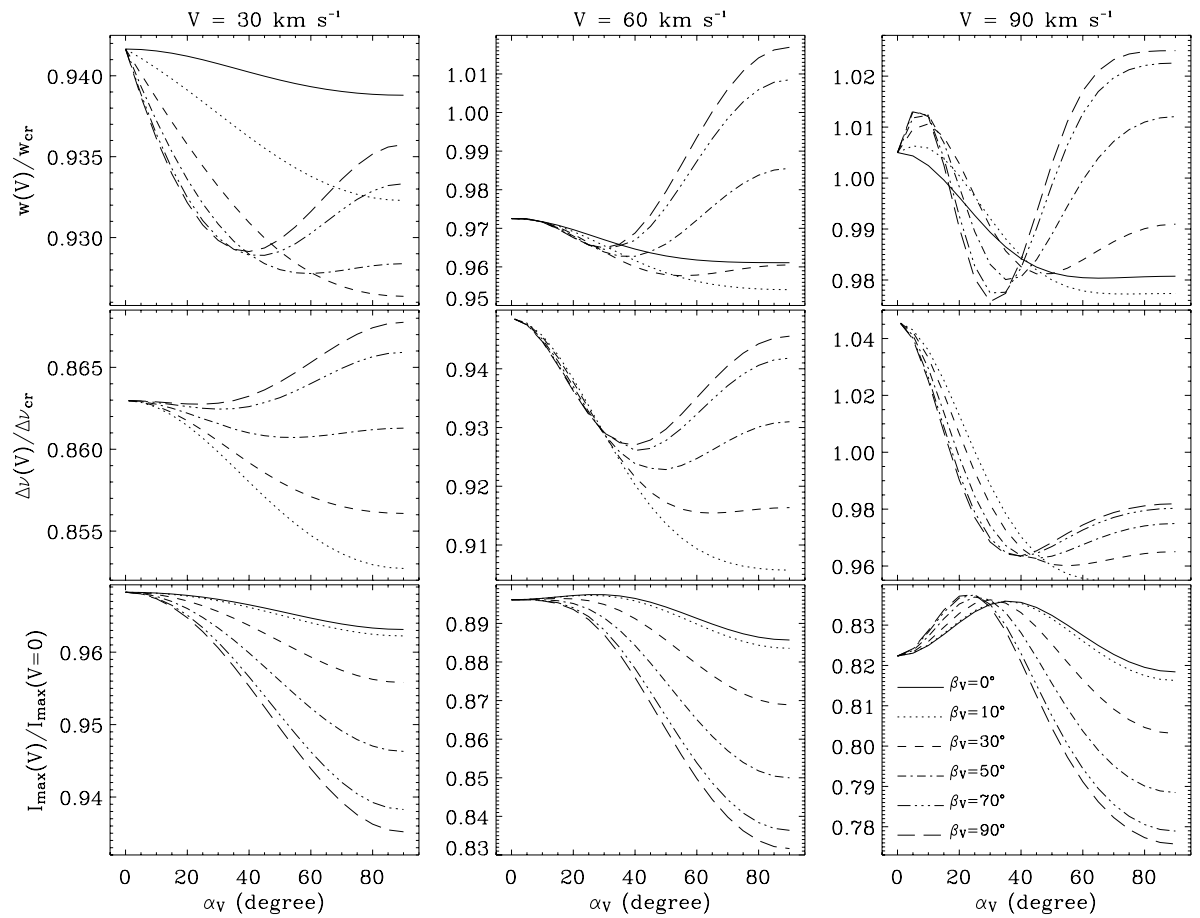

Fig. 8. Relative variation of the Doppler width $w(\boldsymbol{V})$ (top panels) and shift (middle panels) with respect to those obtained for the complete redistribution case for different velocity field vectors $V\left(V, \alpha_{\mathrm{v}}, \beta_{\mathrm{v}}\right)$ of the scattering ions as a function of the polar angle $\alpha_{\mathrm{v}}$ and for different values of the azimuthal angle $\beta_{\mathrm{v}} . \Delta v_{D_{\mathrm{s}}}$ is the width of the velocity field distribution of the scattering ions and also the re-emitted line width $w_{\mathrm{cr}}$ in the complete redistribution approximation. In the present case, $\Delta v_{D_{\mathrm{s}}}=55 \mathrm{~km} \mathrm{~s}^{-1}$. $\Delta v$ is the Doppler shift of the re-emitted line and $d_{\mathrm{cr}}=\Delta v_{Z}=v_{0} V_{Z} / c$ is the shift in the complete redistribution approximation. The bottom panels display the variation of the Doppler dimming. These results are obtained for a heliocentric distance of $1.05 R_{\odot}$.
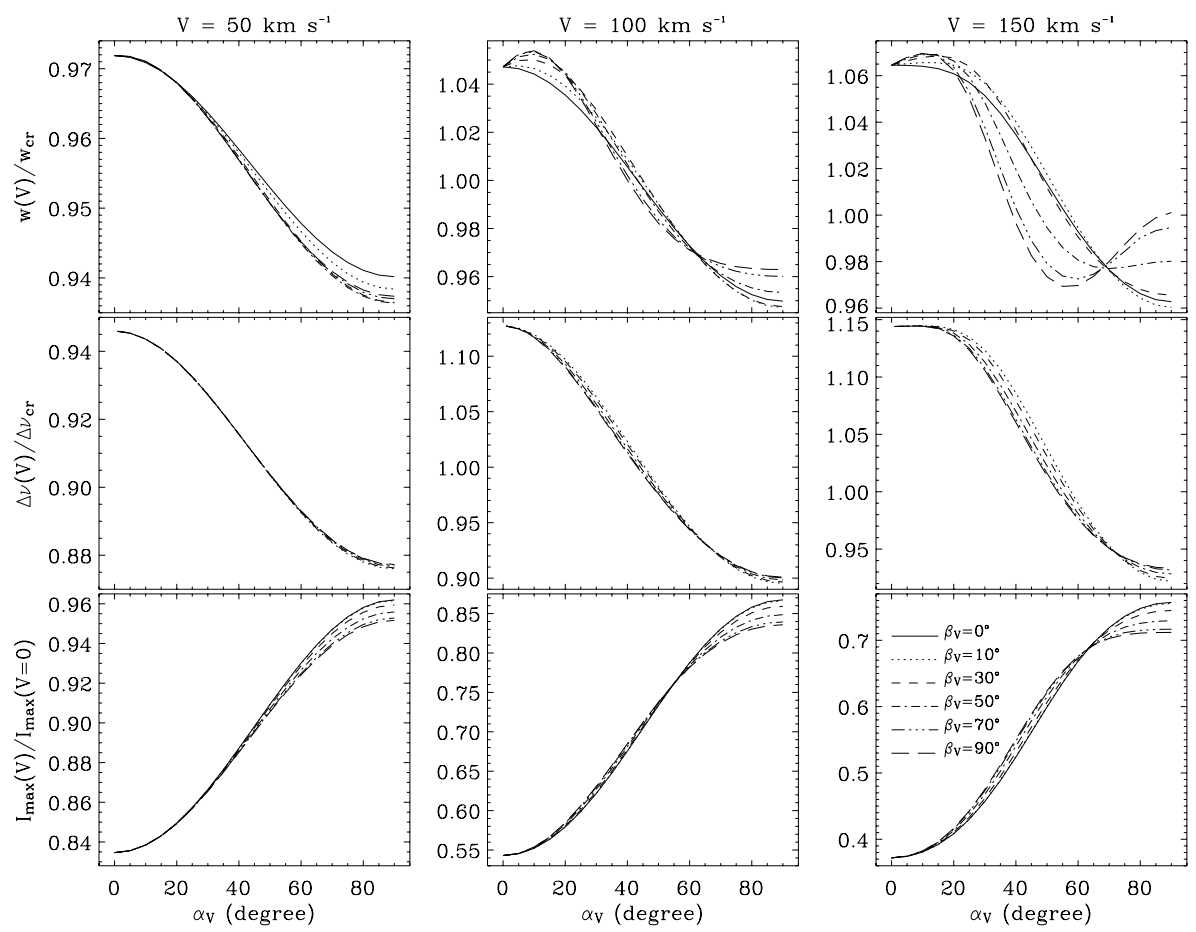

Fig. 9. Same plots as those of Fig. 8 but for a heliocentric distance of $1.5 R_{\odot}$. The variation of the re-emitted line width and the Doppler shift as a function of the polar angle $\alpha_{\mathrm{v}}$ and the azimuthal angle $\beta_{\mathrm{v}}$ is quite different from that given by weaker outflow speeds. The variation of the Doppler dimming is, however, analogous.

outflow speeds the relative line width values are lower than those obtained in the complete Doppler redistribution approximation $\Delta v_{D_{\mathrm{s}}}$. The shift of the radiative profile is also smaller than $\Delta v_{Z}$, and the difference between this shift and $\Delta v_{Z}$ increases with the polar angle. This explains the decrease in the shift ratio $\Delta v(\boldsymbol{V}) / \Delta v_{Z}$ with the polar angle. Since the major part of the incident radiation comes from the solar limb at very small distances such as $1.05 R_{\odot}$, the dimming is more efficient for high values of the polar and azimuth angles. All these facts can explain the increase in the widths and shifts for these angles and the decrease of the dimming $I_{\max }(\boldsymbol{V}) / I_{\max }(\boldsymbol{V}=0)$ with the polar angle, as well as with the azimuth angle.

For strong outflow velocities, the radiative profile is shifted more than the collisional one for low values of the polar angle (top-right panel of Fig. 2). This shift increases over a small range of $\alpha_{\mathrm{v}}$ and begins to decrease after a given value of $\alpha_{\mathrm{v}}$ for a given value of $\beta_{\mathrm{v}}$. The variation of the shift is greater for high values of the azimuth angle. The variation of the shift of the radiative component explains the variation of relative width and shift given by Fig. 8 for $V=90 \mathrm{~km} \mathrm{~s}^{-1}$. 

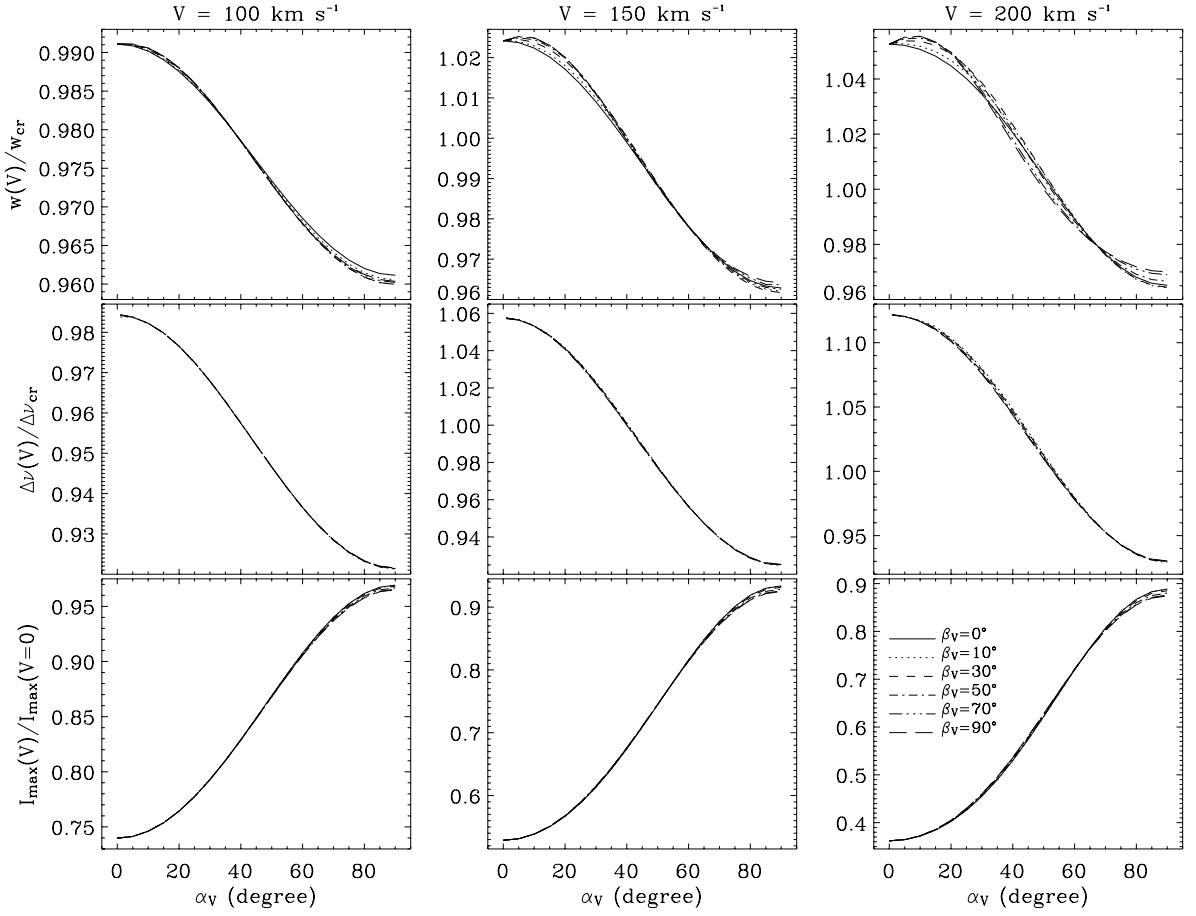

Fig. 10. Same plots as those of Fig. 8 but for a heliocentric distance of $2.0 R_{\odot}$.
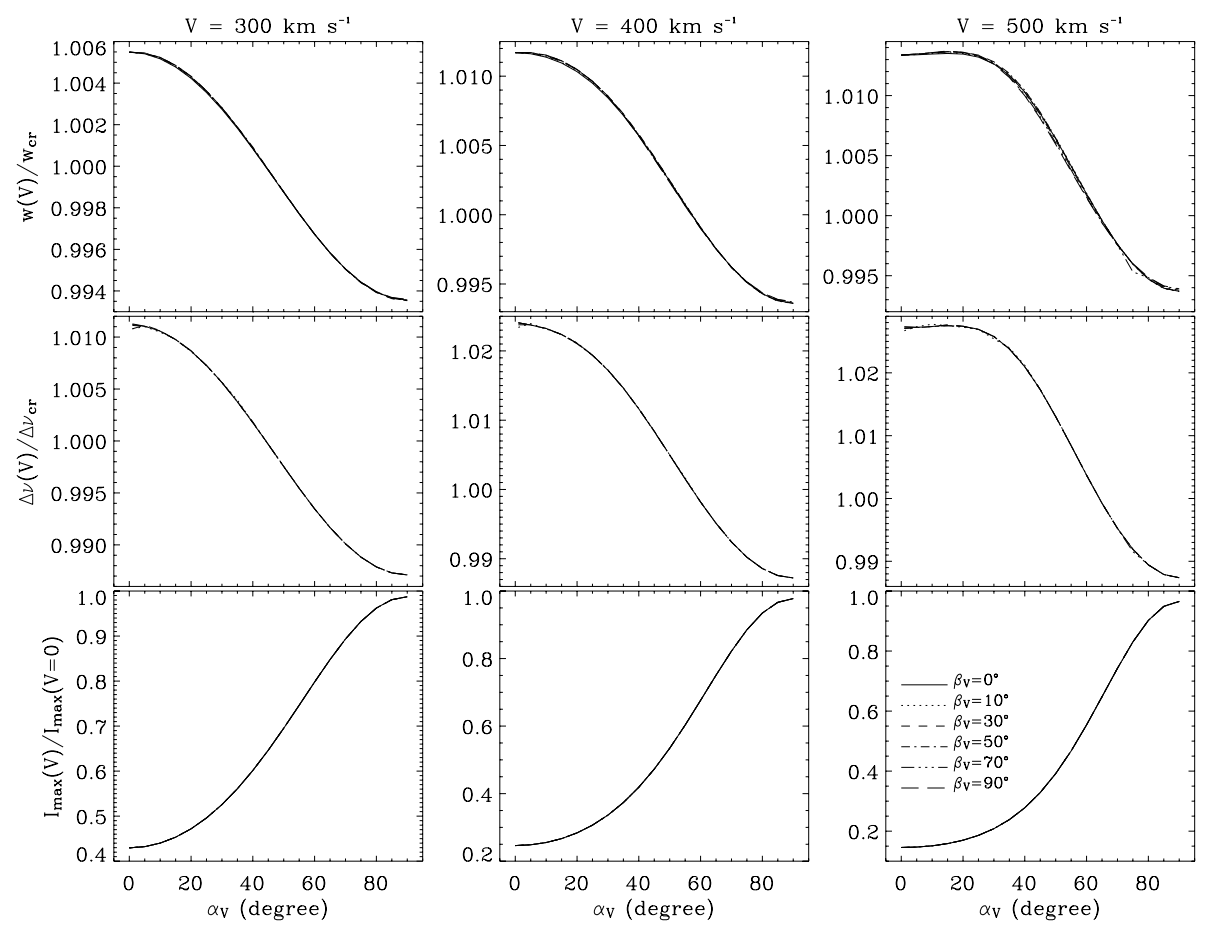

Fig. 11. Same plots as those of Fig. 8 but for a heliocentric distance of $5.0 R_{\odot}$.

\subsubsection{Case of medium and high altitudes: $r=1.5,2.0$ and $5.0 R_{\odot}$}

Figures 9-11 display the same parameters as in Fig. 8. These parameters are obtained at heliocentric distances of 1.5, 2.0, and $5.0 R_{\odot}$. The variation of all the calculated quantities with the azimuth angle $\beta_{\mathrm{v}}$ is much less than in the case of $R=$ $1.05 R_{\odot}$. All these quantities decrease with the polar angle, except for the width at $1.5 R_{\odot}$, where the variation is similar to the one obtained at $1.05 R_{\odot}$. The relative width, shift, and dimming increase with the outflow speed of the scattering ions.

That can explained as follows: the profile of the collisional component has the same properties (width and shift) as in the case of $R=1.05 R_{\odot}$ (dashed lines in Fig. 3). However, the line width of the radiative profile decreases with the polar angle for a given value of the azimuthal angle. Since the shifts are not very high for low values of the outflow speed, we obtain a decreasing behavior with the polar angle of the ratio $\left(w(\boldsymbol{V}) / w_{\mathrm{cr}}\right)$. For low outflows, the shift of the radiative profile is smaller 

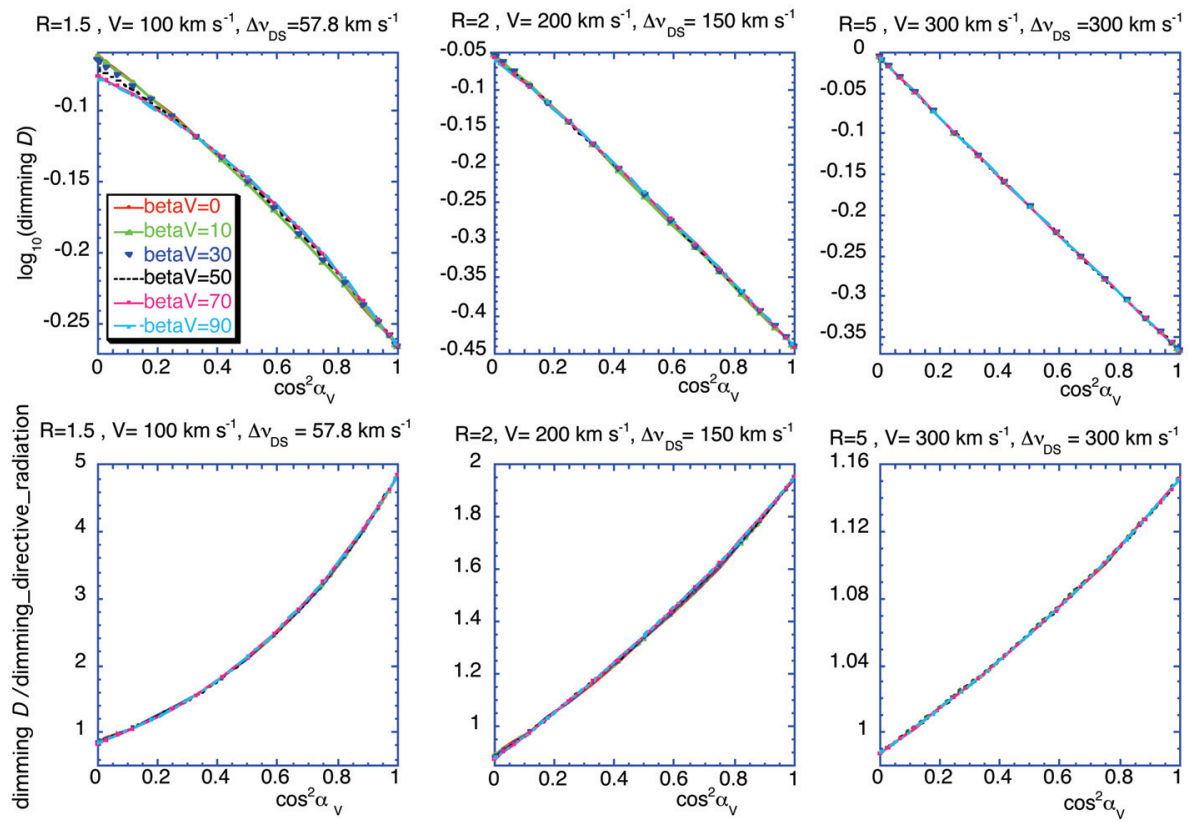

Fig. 12. Angular behavior of the dimming $D$ and comparison with the one obtained with a vertical directive radiation beam. Top panel: logarithm of the dimming for heliocentric distances of $1.5 R_{\odot}, 2.0 R_{\odot}$, and $5.0 R_{\odot}$ versus $\cos ^{2} \alpha_{\mathrm{v}}$. Bottom panel: Ratio of dimming $D$ to the dimming obtained for a vertical directive radiation, for heliocentric distances of $1.5 R_{\odot}, 2.0 R_{\odot}$, and $5.0 R_{\odot}$ versus $\cos ^{2} \alpha_{\mathrm{v}}$. The different series of $\beta_{\mathrm{v}}$ values (from 0 to $90^{\circ}$ ) give quite identical curves for all the panels, except for the top-left one. than that of the collisional profile. The shift difference between these two components increases with the polar angle and hardly changes with the azimuthal angle of the outflow velocity vector. This explains the variation of the shifts shown by the middle panel in Fig. 8 for $V=30 \mathrm{~km} \mathrm{~s}^{-1}$. For higher outflow speeds, the shift of the radiative component is larger than that of the collisional one for low values of $\alpha_{\mathrm{v}}$ (see top-left panel of Fig. 3). In fact, it increases first with the polar angle until a certain limit beyond which it begins to decrease while the polar angle is increasing (see Fig. 3). This explains the variation of the different parameters of the re-emitted line profile in a similar way as for $1.05 R_{\odot}$ : width, shift, and dimming.

At medium and large heliocentric distances, as shown by Fig. 12, the dimming is not very sensitive to the azimuth and depends almost only on the radial component of the drift velocity field. At $R=2 R_{\odot}$ and for higher heliocentric distances, it varies as $\exp \left(-V_{z}^{2}\right)$ (top panel). Moreover, at $R=5 R_{\odot}$, the dimming resulting from a purely directive incident radiation is almost obtained (bottom-right panel). This can be explained as follows: for radial velocities $\alpha_{\mathrm{v}}=0^{\circ}$ and high velocities $\left(300 \mathrm{~km} \mathrm{~s}^{-1}\right)$, the Doppler dimming is important for radiation coming from the vertical, thus the radiative contribution of the limb is not completely negligible and the dimming differs by almost $16 \%$ from the perfectly directive case. For nearly horizontal velocities $\left(\alpha_{\mathrm{v}}=90^{\circ}\right)$, the Doppler dimming has a weaker effect on the radial radiative contribution, than on the the radiation coming from the limb, and the overall dimming differs by less than $1 \%$ from the one obtained for a purely directive incident radiation and is thus only sensitive to the radial component of the velocity field $V_{z}$.

By taking into consideration the accuracy of the measurements and the small change of the line width with the velocity field coordinates, it can also be concluded that the line width will probably be not very useful for the vector velocity diagnostic. In the same way, the shift will be a probe of the line of sight velocity component only.

\section{Conclusion}

These results show that interpretation of the linear polarization parameters, together with the profile of the re-emitted line (especially the Doppler shift and dimming), can yield valuable information about the the outflow velocity vector of the coronal plasma in the acceleration zone of the solar wind. Bearing in mind that the polarization parameters can also be sensitive to the magnetic field via the Hanle effect $\left(\mathrm{P}_{\mathrm{II}}\right), 6$ unknowns will have to be determined, which would be reduced to 4 , since the velocity and the magnetic field are usually assumed to be parallel (or antiparallel) in the solar corona. Consequently, 6 pieces of independent observational data must be obtained. The shift will give the line of sight component of the velocity field, and the dimming give the radial component, while interpretation of the two linear polarization parameters should complete the vectorial diagnostic of the velocity and magnetic field. See the pioneering work on this subject by Raouafi et al. (2002a). In addition, the intensity ratio of different spectral lines (i.e. the 1032-1037 A O vi doublet; Si VIII; etc.) can be used to determine other quantities (i.e. electron densities, electron, and ion temperatures; etc.). See Wilhelm et al. (1998); Doyle et al. (1999); Zangrilli et al. (2002); Gabriel et al. (2003). These quantities are necessary for interpretation of the polarization and line profiles in terms of velocity vectors. However, it is also important to take the correct anisotropy of the incident radiation into account (center-to-limb brightening, plumes, and inter-plumes structures, shape of the coronal polar hole, etc.).

We conclude that the OVI line at $1032 \AA$ is a good candidate for performing the diagnostic of the solar wind outflow velocity in the solar wind acceleration region. On one hand, at large distances from the limb, the results show that the Doppler width, shift, dimming, and polarization parameters of the scattered O VI $1032 \AA$ are not very sensitive to the azimuth angle of the velocity field. Then the results of the complete redistribution approximation are obtained. The radial component of the velocity field can be obtained through to the interpretation 
of the dimming, and the component along the line of sight can be obtained through the shift. The component of the velocity field along the perpendicular to the scattering plane cannot be obtained with this method. At medium-large distances, the ensemble of 4 parameters shift, dimming, and the two linear polarization parameters become sensitive to the three components of the velocity field; but the accuracy of the complete vectorial diagnostic will remain rather imprecise. On the other hand, at small and medium distances where the solar wind accelerates, all these parameters are sensitive to the three components of the velocity field vector in a different manner. Thus a complete vector velocity field diagnostic would be achieved with rather good accuracy. See the pioneering constraints obtained by Raouafi et al. (2002a,b) limited by accuracy of the measurements. The present work demonstrates the importance of accurate polarization parameters measurements in the EUV for coronal diagnostics of vector velocity and magnetic field vectors. We finally conclude that our proposed method is realistic.

In addition, we notice that the integration along the line of sight has a significant effect on the spectral line profiles emitted in the solar corona (Raouafi \& Solanki 2004, 2006), which could be very important for the resulting polarization of the scattered line. This has to be taken into account for a realistic interpretation of coronal observations. This consideration, general to all spectroscopic diagnostics of optically thin lines, is essential for spectropolarimetric diagnostics where angular processes are of drastic importance.

Acknowledgements. The authors would like to thank the referee for the detailed comments and meaningful suggestions that greatly improved the paper. They would also like to thank Dr. V. Bommier for useful suggestions and comments. The NSO is operated by the Association of Universities for Research in Astronomy (AURA), Inc. under cooperative agreement with the National Science Foundation (NSF). N.-E. Raouafi's work is supported by NSO and the NASA grant NNH05AA12I.

\section{References}

Blum, K. 1981, Density Matrix Theory and Applications (New York: Plenum Press)

Cohen-Tannoudji, C. 1977, Atoms in Strong Resonant Fields, ed. R. Balian, S. Haroche, \& S. Liberman, Frontiers in Laser Spectroscopy, Les Houches 1975 (North-Holland, Amsterdam)

Cohen-Tannoudji, C., Dupont-Roc, J., \& Grynberg, G. 1988, Processus d'Interaction entre Photons et Atomes (Paris: Inter Editions/ Editions du CNRS)

Domingo, V., Fleck, B., \& Poland, A. I. 1995, Sol. Phys., 162, 1

Doyle, J. G., Teriaca, L., \& Banerjee, D. 1999, A\&A, 349, 956

Gabriel, A. H. 1971, Sol. Phys., 21, 392

Gabriel, A. H., Bely-Dubau, F., \& Lemaire, P. 2003, ApJ, 589, 623

Harrison, R. A., Sawyer, E. C., Carter, M. K., et al. 1995, Sol. Phys., 162,233

Harrison, R. A., Fludra, A., Pike, C. D., et al. 1997, Sol. Phys., 170, 123
Hummer, D. 1962, Stellar Atmospheres, 2nd ed. (Freeman \& Co) Hyder, C. L., \& Lites, B. W. 1970, Sol. Phys., 14, 147

Kohl, J. L., \& Withbroe, G. L. 1982, ApJ, 256, 253

Kohl, J. L., Esser, R., Gardner, L. D., et al. 1995, Sol. Phys., 162, 313

Kohl, J. L., Noci, G., Antonucci, E., et al. 1997, Sol. Phys., 175, 613

Kohl, J. L., Esser, R., Cranmer, S. R., et al. 1999, ApJ, 510, L59

Lamy, P., Quemerais, E., Liebaria, A., et al. 1997, Fifth SOHO Workshop, ESA SP-404, 491

Landi Degl'Innocenti, E., \& Landolfi, M. 2004, Polarization in Spectral Lines (Kluwer Acad. Publ.)

Lemaire, P., Wilhelm, K., Curdt, W., et al. 1997, Sol. Phys., 170, 105

Mihalas, D. 1978, MNRAS, 125, 21

Mitchell, A. C. G., \& Zemansky, M. W. 1934, Resonance Radiation and Excited Atoms (Cambridge University Press)

Oxenius, J. 1986, Kinetic Theory of Particles and Photons (SpringerVerlag)

Porter, J. G., West, E. A., Davis, J. M., et al. 2003, BAAS, 35, American Astronomical Society, SPD meeting 34

Raouafi, N.-E., Lemaire, P., \& Sahal-Bréchot, S. 1999, A\&A, 345, 999

Raouafi, N.-E., Sahal-Bréchot, S., Lemaire, P., \& Bommier, V. 2002a, A\&A, 390, 691

Raouafi, N.-E., Sahal-Bréchot, S., \& Lemaire, P. 2002b, A\&A, 396, 1019

Raouafi, N.-E., \& Solanki, S. K. 2003, A\&A, 412, 271

Raouafi, N.-E., \& Solanki, S. K. 2004, A\&A, 427, 725

Raouafi, N.-E., \& Solanki, S. K. 2006, A\&A, 445, 735

Rompolt, B. 1967, Acta Astron., 17, 329

Rompolt, B. 1969, IV Consultation on Solar Physics and Hydromagnetics, Proceedings of the conference held 20-23 September, 1966 in Sopot, ed. by Jan Megentaler, A Universitatis Wratislaviensis No. 77. Matematyka, Fizyka, Astronomia VIII, Wroclaw, p.117

Sahal-Bréchot, S., Malinovsky, M., \& Bommier, V. 1986, A\&A, 168, $284\left(\mathrm{P}_{\mathrm{I}}\right)$

Sahal-Bréchot, S., Feautrier, N., Bommier, V., \& de Kertanguy, A. 1992, Proc. ESA Workshop on Solar Physics and Astrophysics at Interferometric Resolution (SIMURIS). Paris (France), 1992 February 17-19, ed. L. Damé, \& T. D. Guyenne, ESA SP344,81

Sahal-Bréchot, S., \& Choucq-Bruston, M. 1994, "La polarimétrie outil pour l'étude de l'activité magnétique solaire et stellaire", Atelier du GdR Magnétisme dans les étoiles de type solaire du CNRS, Nice Observatory (France), 1994 November 16-18, ed. N. Mein, \& S. Sahal-Bréchot (Paris Observatory Publ.), 77

Sahal-Bréchot, S., Bommier, V., \& Feautrier, N. 1998, A\&A, 340, $579\left(\mathrm{P}_{\mathrm{II}}\right)$

Thomas, R. J. 2003, BAAS, 34, 641 (AAS 200th meeting, Albuquerque, June 2002)

Vial, J.-C., Song, X., Lemaire, et al. 2003, in Innovative Telescopes and Instrumentation for Solar Astrophysics, ed. S. L. Keil, \& S. V. Avakyan, Proc. SPIE, 4853, 479

Wilhelm, K., Curdt, W., Marsch, E., et al. 1995, Sol. Phys., 162, 189

Wilhelm, K., Lemaire, P., Curdt, W., et al. 1997, Sol. Phys., 170, 75

Wilhelm, K., Marsch, E., Dwivedi, et al. 1998, ApJ, 500, 1023

Zangrilli, L., Poletto, G., Nicolosi, P., Noci, G., \& Romoli, M. 2002, ApJ, 574, 477 